\title{
Determination of Nickel in End-Fitting of Spoolable Reinforced Plastic Line Pipe
}

\author{
Xiaodong Shao ${ }^{1,2}$ * \\ ${ }^{1}$ State Key Laboratory for Performance and Structure Safety of Petroleum Tubular Goods and \\ Equipment Materials, No. 89 Jinyeer Road, Xi'an 710077, China \\ ${ }^{2}$ CNPC Tubular Goods Research Institute, No. 89 Jinyeer Road, Xi'an 710077, China
}

\begin{abstract}
Spoolable reinforced plastic line pipe will be widely used in the exploitation of acid gas and oil wells. End-fitting is a mechanical device that forms the transition from the spoolable reinforced plastic line pipe to the connector. It was well known that nickel was an important element in the end-fitting. In this paper, a novel spectrophotometric method was described for determination of nickel in the end-fitting of spoolable reinforced plastic line pipe. The method was based on the color reaction between nickel and dimethylglyoxime, which was favored in an alkaline medium, producing a red compound in the presence of ammonium persulfate as an oxidant. Under the optimum reaction conditions the absorption value was proportional to the concentration of nickel in the range of $0.080 \sim 0.50 \%(\mathrm{R} 2=0.9999)$, and the relative standard deviation was less than $3.0 \%(n=5)$. The proposed method was applied successfully to determine nickel in end-fitting of spoolable reinforced plastic line pipe.
\end{abstract}

\section{Introduction}

The condition of gas and oil wells in the west of China has complex characteristics such as deep, high pressure, and serious corrosive that only corrosion-resistant pipes can be applied to explore. One key feature there is obvious existence of corrosive hydrogen sulfide medium. In order to meet the exploitation requirements of these oil-gas wells with high pressure and high partial pressure of $\mathrm{H}_{2} \mathrm{~S}$ and $\mathrm{CO}_{2}$, the pipes must adopt new materials with excellent resistance to corrosion. Spoolable reinforced plastic line pipe (Fig. 1), also known as

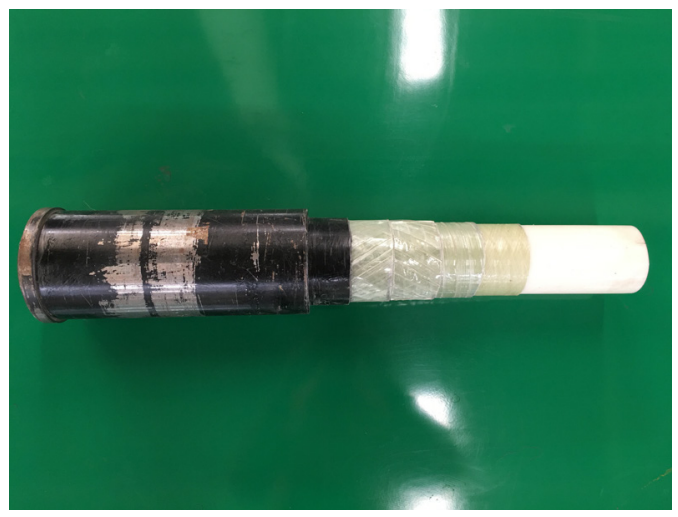

Fig. 1. Spoolable reinforced plastic line pipe

* Corresponding author: shaoxd@163.com 
flexible composite pipes or reinforced thermoplastic pipes (RTP), contain three layers, among which, neck bush and reinforcement layer are keys to determine mechanical and thermal properties of these pipes. These composite pipes are key to enabling the industry to meet some of the energy security challenges that nations face today. The ability to combine tailored strength with flexibility, low weight, high thermal and pressure tolerance, minimal flow resistance, high resilience and durability in large-bore pipes enables them to surpass the capabilities of current predominantly metal piping-albeit often polymer lined. The spoolable reinforced plastic line pipe has excellent resistance to corrosion. For example, glass fibre reinforced polyoxymethylene and polypropylene (PP) performed well in saltwater, while carbon fibre reinforced polyetheretherketone proved well suited to a sour oil environment [1]. The fact that spoolable reinforced plastic line pipe is more flexible and spoolable than steel, and is also much lighter, means that it can be carried and deployed from smaller vessels and managed more easily. End-fitting is a mechanical device that forms the transition from the spoolable reinforced plastic line pipe to the connector. In order to control the quality of end-fitting products, the contents of trace impurities and alloy elements in end-fitting should be determined. It is well known that nickel is an important element in end-fitting. Therefore, a rapid and precise analytical method for the determination of nickel is essentially required for the production control of spoolable reinforced plastic line pipe. Numerous analytical methods were employed for the determination of nickel in different types of matrices including atomic absorption spectrometry [2,3], atomic emission spectrophotometry [4], molecular fluorescence spectroscopy [5], electroanalytical method [6] and spectrophotometry [7,8]. Spectrophotometric methods occupy special position due to their simplicity, less expensive instrumentation and high sensitivity. Similarly, spectrophotometric methods also play an important role in nickel determination. This present method was based on the color reaction between nickel and dimethylglyoxime, which was favored in an alkaline medium, producing a red compound in the presence of ammonium persulfate as an oxidant. The characteristic wavelength of maximum absorption was measured at $530 \mathrm{~nm}$. Under the optimum reaction conditions the absorption value (A) was proportional to the concentration of nickel in the range of $0.080 \% \sim 0.50 \%\left(\mathrm{R}^{2}=0.9999\right.$, mass fraction $)$, and the relative standard deviation (RSD) was less than $3.0 \%(n=5)$. The proposed method was free from the interference from a large number of analytical important elements and has been applied satisfactorily to the determination of nickel in end-fitting of spoolable reinforced plastic line pipe samples with the improved accuracy and precision, respectively. Results were in agreement with those attained by inductively coupled plasma atomic emission spectrophotometry at the $95 \%$ confidence level. The satisfactory performance in the determination of nickel in end-fitting of spoolable reinforced plastic line pipe demonstrated that the method was practical and suitable not only for quality control analysis but also for product analysis, confirming the promise for spoolable reinforced plastic line pipe research.

\section{Experiment}

\subsection{Apparatus and reagents}

A UV-visible spectrophotometer (Model TU-1901, Beijing Persee General Instrument Co. Ltd.) with 2.0-cm quartz cells was employed for all absorbance measurements.

All chemicals including perchloric acid (Tianjin Tianda Reagent Plant), nitric acid (Xi'an Chemical Reagent Plant), ethanol (Tianjin Dongfang Reagent Plant) and hydrochloric acid (Sichuan Xilong Chemical Co. Ltd.) used in the experiments were of analytical reagent grade and were used as received. Solutions of ammonium persulfate 
(Tianjin Tianda Reagent Plant), natrium tartrate (Xi'an Chemical Reagent Plant) and sodium hydroxide (Institute of Shanghai Fine Chemical Materials) were prepared daily by dissolving the reagents in deionised water that treated with a Milli-Q water purification system (Millipore, Bedford, MA, USA). Solution of dimethylglyoxime (Xi'an Chemical Reagent Plant) were prepared dissolving the reagents in ethanol, and the solutions of nickel were prepared from the steel certified reference materials (CRM) including GBW 01301, GBW 01302, GBW 01304, GBW 01306, GBW 454 (Institute of Anshan Iron and Steel Group Corporation), GBW 01354 (Institute of Fushun Iron and Steel Group Corporation). All working strength solutions in the experiment were prepared with deionised water.

\subsection{General procedures}

Weigh accurately $0.2000 \mathrm{~g}$ of drilled low alloy steel samples and dissolve the samples in 10 $\mathrm{mL}$ mixture acid solution of hydrochloric acid $25 \%(\mathrm{~V} / \mathrm{V})$ and nitric acid $25 \%(\mathrm{~V} / \mathrm{V})$ in a $150-\mathrm{mL}$ taper bottle. Continue the treatment with mixture acid till the sample goes into solution. Add $5.0 \mathrm{~mL}$ perchloric acid $\left(\rho 1.67 \mathrm{~g} \cdot \mathrm{mL}^{-1}\right)$ and heat to fumes of perchloric acid, and evaporate almost to dryness. Cool the solution, add $50 \mathrm{~mL}$ deionised water to dissolve some salts, and cool the solution at room temperature. Finally, dilute the solution in a 100$\mathrm{mL}$ volumetric flask, followed make up to the mark with deionised water, mix well and let stand. Then, transfer the above stock solution $10.0 \mathrm{~mL}$ in a $50-\mathrm{mL}$ volumetric flask, add 10 $\mathrm{mL}$ of $300.0 \mathrm{mg} \cdot \mathrm{mL}^{-1}$ solution of natrium tartrate, $10.0 \mathrm{~mL}$ of $100.0 \mathrm{mg} \cdot \mathrm{mL}^{-1}$ solution of sodium hydroxide, $2.0 \mathrm{~mL}$ of $10.0 \mathrm{mg} \cdot \mathrm{mL}^{-1}$ solution of dimethylglyoxime $5 \mathrm{~mL}$ of 40.0 $\mathrm{mg} \cdot \mathrm{mL}^{-1}$ solution of ammonium persulfate, followed make up to the mark with deionised water, mix well and let stand. Then, transfer the above stock solution in $2.0-\mathrm{cm}$ quartz cells, this solution was the work solution. At the same time, transfer the first stock solution 10.0 $\mathrm{mL}$ in a $50-\mathrm{mL}$ volumetric flask, add $10 \mathrm{~mL}$ of $300.0 \mathrm{mg} \cdot \mathrm{mL}^{-1}$ solution of natrium tartrate, $10.0 \mathrm{~mL}$ of $100.0 \mathrm{mg} \cdot \mathrm{mL}^{-1}$ solution of sodium hydroxide, $2.0 \mathrm{~mL}$ of $95 \%(\mathrm{~V} / \mathrm{V})$ ethanol、 5 $\mathrm{mL}$ of $40.0 \mathrm{mg} \cdot \mathrm{mL}^{-1}$ solution of ammonium persulfate, followed make up to the mark with deionised water, mix well and let stand. Then, transfer the above stock solution in $2.0-\mathrm{cm}$ quartz cells, this solution was employed as the blank solution in subsequent experiments. Measure the absorbance at $530 \mathrm{~nm}$ using a UV-visible spectrophotometer with $2.0-\mathrm{cm}$ quartz cells against a reagent blank prepared similarly. Plot the amount of nickel in the sample solution against absorbance to obtain the calibration graph.

\section{Results and discussion}

\subsection{Spectral characteristics}

The absorption spectra of the reagents blank, nickel against the reagent blank were tested from $400 \mathrm{~nm}$ to $700 \mathrm{~nm}$. The nickel yields the characteristic wavelength of maximum absorption at $530 \mathrm{~nm}$ wavelength. However, the absorption spectrum of the reagents blank under similar conditions shown that it does not absorb at $530 \mathrm{~nm}$. And the experimentation also demonstrated that nickel increases the absorbance value considerably, resulting in increased sensitivity of the method. Therefore, measure the absorbance of nickel at $530 \mathrm{~nm}$.

\subsection{Select dissolved acid for low alloy steel samples}

The effect of the different acids for dissolve the low alloy steel samples was examined. It was found that a solution mixed with $25 \%(\mathrm{~V} / \mathrm{V})$ hydrochloric acid and $25 \%(\mathrm{~V} / \mathrm{V})$ nitric acid has a good effect for dissolve the low alloy steel samples. Thus, an acid solution mixed 
with $25 \%(\mathrm{~V} / \mathrm{V})$ hydrochloric acid and $25 \%(\mathrm{~V} / \mathrm{V})$ nitric acid was recommended for dissolve the low alloy steel samples in subsequent studies.

\subsection{Influence of the amounts of chromogenic reagent}

Dimethylglyoxime was selected as the chromogenic reagent in the proposed method. The effect of the chromogenic reagent was examined by measuring the absorbance of solution containing certain amounts of nickel and variable amounts of dimethylglyoxime. It was found that $2.0 \mathrm{ml}$ of $10.0 \mathrm{mg} \cdot \mathrm{mL}^{-1}$ dimethylglyoxime solution sufficed to complex the amounts of nickel taken; with higher concentrations the absorbance was essentially constant. Two milliliters of $10.0 \mathrm{mg} \cdot \mathrm{mL}^{-1}$ dimethylglyoxime solution were recommended as a suitable amount of chromogenic reagent.

\subsection{Influence of the amounts of oxidant}

Ammonium persulfate was selected as the oxidant in the proposed method. The effect of the oxidant was examined by measuring the absorbance of solution containing certain amounts of nickel and variable amounts of ammonium persulfate. It was found that $5.0 \mathrm{~mL}$ of $40.0 \mathrm{mg} \cdot \mathrm{mL}^{-1}$ solution of ammonium persulfate sufficed to oxidate the amounts of nickel taken, with higher concentrations the absorbance was essentially constant. Five milliliters of $40.0 \mathrm{mg} \cdot \mathrm{mL}^{-1}$ ammonium persulfate solution were recommended as a suitable amount of oxidant.

\subsection{Stoichiometry and stability constant}

The stoichiometry of the nickel complex with dimethylglyoxime in solution was studied spectrophotometrically by Job's method of continuous variation and equilibrium shift method. The results indicate that the molar ratio of nickel to dimethylglyoxime is $1: 4$. The molar extinction coefficient $(\varepsilon)$ of the ligand complex is $1.3 \times 10^{4} \mathrm{~L} \cdot \mathrm{mol}^{-1} \cdot \mathrm{cm}^{-1}$, which calculated from these results following the described method.

\subsection{Interference studies}

The extent of interference by diverse ions was determined by measuring the absorbance of solutions containing nickel and various amounts of diverse ions. The criterion for interference was an absorbance value varying by more than $+2 \%$ from the expected value of nickel alone. The results show that a large excess of cations and anions which are usually associated in the determination of nickel do not interfere. The colorless metal ions do not interfere. The tolerable amounts of foreign species with respect to nickel for interference at $+2 \%$ level were over 200 300 for the colored metal ions. When the colored metal ions contents of not more than 200 300 times nickel, it can be in some color solution adding a few drops of EDTA solution or sodium nitrite solution to fade the color, this solution as the measured reference solution to eliminate the interference. There was not obvious interference ions exist in end-fitting of spoolable reinforced plastic line pipe.

\subsection{Preparation of the calibration graph}

Under the optimal conditions, a series of standard solutions of nickel were prepared by the different steel certified reference materials in a $100-\mathrm{mL}$ volumetric flask. add $10 \mathrm{~mL}$ of $300.0 \mathrm{mg} \cdot \mathrm{mL}^{-1}$ solution of natrium tartrate, $10.0 \mathrm{~mL}$ of $100.0 \mathrm{mg} \cdot \mathrm{mL}^{-1}$ solution of sodium 
hydroxide, $2.0 \mathrm{~mL}$ of $10.0 \mathrm{mg} \cdot \mathrm{mL}^{-1}$ solution of dimethylglyoxime $5 \mathrm{~mL}$ of $40.0 \mathrm{mg} \cdot \mathrm{mL}^{-1}$ solution of ammonium persulfate, followed make up to the mark with deionised water, mix well and let stand. Then, transfer the above stock solution in $2.0-\mathrm{cm}$ quartz cells, this solution was the work solution. At the same time, transfer the first stock solution $10.0 \mathrm{~mL}$ in a $50-\mathrm{mL}$ volumetric flask, add $10 \mathrm{~mL}$ of $300.0 \mathrm{mg} \cdot \mathrm{mL}^{-1}$ solution of natrium tartrate, 10.0 $\mathrm{mL}$ of $100.0 \mathrm{mg} \cdot \mathrm{mL}^{-1}$ solution of sodium hydroxide, $2.0 \mathrm{~mL}$ of $95 \%(\mathrm{~V} / \mathrm{V})$ ethanol $5 \mathrm{~mL}$ of $40.0 \mathrm{mg} \cdot \mathrm{mL}^{-1}$ solution of ammonium persulfate, followed make up to the mark with deionised water, mix well and let stand. Measure the absorbance at $530 \mathrm{~nm}$ using a UVvisible spectrophotometer with $2.0-\mathrm{cm}$ quartz cells against a reagent blank. The absorbance of the solution was proportional to the concentration of nickel in the range of $0.080 \% \sim 0.50 \%$ (mass fraction). Plot the amount of nickel in the sample solution against absorbance to obtain the calibration graph. The linear regression equation for nickel was $\mathrm{A}$ $=1.20752 \mathrm{C}+0.00198, \mathrm{R}^{2}=0.9999$, and the relative standard deviation (RSD) was less than $3.0 \%(\mathrm{n}=5)$.

\section{Applications}

\subsection{Determination of nickel in end-fitting of spoolable reinforced plastic line pipe samples}

The recommended procedure has been applied satisfactorily to the determination of nickel in end-fitting of spoolable reinforced plastic line pipe samples. The samples were dissolved according to the following procedure. Measure the absorbance at $530 \mathrm{~nm}$ using a UVvisible spectrophotometer with $2.0-\mathrm{cm}$ quartz cells against a reagent blank. The results were summarized in Table 1 with the good accuracy and precision.

Table 1. Results of determination of nickel in end-fitting samples $(\omega, n=5)$

\begin{tabular}{|c|c|c|c|c|}
\hline \multirow{2}{*}{ Sample No. } & \multirow{2}{*}{$\boldsymbol{A}$} & \multirow{2}{*}{ RSD (\%) } & \multicolumn{2}{|c|}{ Content (\%) } \\
\cline { 3 - 5 } & & 0.68 & Proposed method & AES \\
\hline 1 & 0.4488 & 1.56 & 0.37 & 0.37 \\
\hline 2 & 0.4608 & 2.03 & 0.38 & 0.39 \\
\hline 3 & 0.4488 & 1.21 & 0.37 & 0.37 \\
\hline 4 & 0.4729 & 1.88 & 0.39 & 0.38 \\
\hline 5 & 0.4729 & 0.93 & 0.36 & 0.40 \\
\hline 6 & 0.4367 & 2.54 & 0.12 & 0.36 \\
\hline 7 & 0.1469 & 1.47 & 0.10 & 0.11 \\
\hline 8 & 0.1227 & 2.33 & 0.11 & 0.12 \\
\hline 9 & 0.1348 & 1.64 & 0.13 & 0.13 \\
\hline 10 & 0.1590 & 1.35 & 0.10 & 0.10 \\
\hline 11 & 0.1227 & 1.96 & 0.10 & 0.11 \\
\hline 12 & 0.1227 & 1.01 & 0.20 & 0.19 \\
\hline 13 & 0.2435 & 0.98 & 0.21 & 0.20 \\
\hline 14 & 0.2556 & 1.24 & 0.21 & 0.21 \\
\hline 15 & 0.2556 & 2.18 & 0.24 & 0.25 \\
\hline 16 & 0.2918 & 1.57 & 0.22 & 0.22 \\
\hline 17 & 0.2676 & 1.39 & 0.21 & 0.20 \\
\hline 18 & 0.2556 & & & \\
\hline
\end{tabular}




\section{Conclusions}

Based on the chromogenic reaction between nickel and dimethylglyoxime, a simple and accurate spectrophotometric method for determination of nickel in end-fitting of spoolable reinforced plastic line pipe was proposed. The red colored nickel solution exhibits absorption maximum at $530 \mathrm{~nm}$. The proposed method was free from the interference from a large number of analytical important elements and has been applied satisfactorily to the determination of nickel in end-fitting of spoolable reinforced plastic line pipe samples. Compared with other methods for the determination of nickel, this method offers advantages of simplicity, less expensive instrumentation and high efficient. The satisfactory performance in the determination of nickel in end-fitting of spoolable reinforced plastic line pipe demonstrated that the method was practical and suitable not only for quality control analysis but also for product analysis, confirming the promise for spoolable reinforced plastic line pipe research.

The authors gratefully acknowledge the State Key Laboratory for Performance and Structure Safety of Petroleum Tubular Goods and Equipment Materials, and CNPC Tubular Goods Research Institute.

\section{References}

1. K. Yu, E.V. Morozov, M.A. Ashraf, K. Shankar, Composite Structures 131, 453 (2015)

2. M. Pozzatti, B. Welz, Microchemical Journal 133, 162 (2017)

3. B.M. Soares, R.F. Santos, F.A. Duarte, Talanta 160, 454 (2016)

4. L. Li, H. Gao, J. Zhang, Metallurgical Analysis 27, 41 (2007)

5. M. C. Talio, M. Alesso, M. Acosta, V. S. Wills, L. P. Fernández, Talanta 174, 221 (2017)

6. E. Punrat, P. Tutiyasarn, O. Chailapakul, Talanta 168, 286 (2017)

7. B.N. Kumar, S. Kanchi, M.I. Sabela, K. Bisetty, Karbala International Journal of Modern Science 2, 239 (2016)

8. Y. Guo, H. Zhao, Q. Zhang, X. Bian, Spectrochimica Acta Part A 173, 532 (2017) 